\title{
A dor que não pode calar: reflexões sobre o luto em tempos de Covid-19
}

\author{
Fernanda Gomes Lopes ${ }^{\mathrm{a}}$ \\ Maria Juliana Vieira Lima ${ }^{a}$ (e \\ Rebecca Holanda Arrais ${ }^{\mathrm{b}} \odot$ \\ Natália Dantas do Amaral` ${ }^{\circledR}$ \\ a Instituto Escutha, Fortaleza, CE, Brasil \\ ${ }^{b}$ Centro Universitário Christus, Fortaleza, CE, Brasil \\ c Universidade de São Paulo, São Paulo, SP, Brasil
}

\begin{abstract}
Resumo: A Covid-19 provocou número elevado de perdas e mudanças nos processos de cuidado e despedida, fatores que podem dificultar a elaboração do luto. Objetivamos, assim, compreender as particularidades do luto no âmbito da Covid-19, ressaltando a importância da adaptação e da criação de estratégias voltadas ao cuidado da saúde mental nos processos de luto. $O$ ensaio realiza um panorama teórico, indicando elementos presentes na pandemia com potencial para interferir no luto, e apresenta um levantamento de iniciativas adaptadas a tal contexto. A pandemia trouxe mudanças na forma de experienciar o morrer. Evidenciam-se iniciativas na tentativa de mitigar os efeitos emocionais, cognitivos e comportamentais. O momento convoca a repensar conceitos e (re)criar compreensões acerca da morte e do luto, ressaltando-se a importância da Rede de Saúde Mental, com o fortalecimento de ações de base sociocomunitária, reconhecimento de diferentes níveis de complexidade das demandas e acesso organizado à atenção especializada.
\end{abstract}

Palavras-chave: luto, pesar, pandemias, impacto psicossocial, infecções por coronavírus.

\section{Introdução}

Na sociedade contemporânea ocidental, a morte é um tabu (Ariès, 1977/2003; Kovács, 2012), "assunto socialmente evitado e politicamente incorreto" (Moritz, 2005, p. 51), por estar associada a inquietações e sentimentos adversos. Sua concepção envolve aspectos culturais, religiosos, filosóficos e científicos, permitindo reconhecer suas variabilidades contextual e temporal. Embora essa associação pareça natural, a visão do morrer modificou-se ao longo dos séculos. Segundo Ariès (1977/2003), "a atitude antiga, em que a morte é simultaneamente familiar, próxima e atenuada, indiferente, opõe-se muito à nossa, em que a morte provoca medo, a ponto de nem ousarmos dizer-lhe o nome" (p. 26).

$\mathrm{O}$ autor descreve que, desde o século XX, mediante avanços tecnocientíficos, a morte é interdita, aproximando-se do lugar de recusa e negação. Com evitação e incômodo associados à sua consciência, tentamos passar despercebidos pela morte, buscando continuar a vida de maneira como se aquela não pudesse ocorrer.

$\mathrm{Na}$ passagem do século XX para o XXI, se reconhece o conceito de morte escancarada, concomitantemente à interdita. Conforme Kovács (2003) trata-se da "morte que invade, ocupa espaços, penetra na vida das pessoas a qualquer hora ... é brusca,

* Endereço para correspondência: fernanda.gomeslopes@hotmail.com repentina, invasiva e involuntária" (p. 142). Destarte, a morte, noticiada nos meios de comunicação ou que insiste em aparecer em fotos e vídeos nas redes sociais, ganha lugar de banalização e afastamento, sendo vista como algo que não nos afeta pessoalmente.

A negação da morte, evidenciada nesses dois processos, distancia o homem da necessidade de contato com o tema, contribuindo para maior dificuldade da sociedade ocidental em lidar com perdas. Contudo destacamos que uma perda significativa pode impactar o indivíduo e afetar seu desenvolvimento. Esse processo de ruptura é chamado luto, conceito complexo que vem sendo definido historicamente por diferentes autores. Nesse primeiro momento, é importante destacar que se refere a um processo "natural e necessário, pois se trata de dolorida elaboração da perda" (Alves \& Kovács, 2016, p. 403) e é vivido de maneira individual e subjetiva, com impacto nas diversas áreas da vida (Parkes, 1998).

Diversos fatores interferem na elaboração do luto, entre eles a circunstância da morte. Assim, "compreender os aspectos relacionados à morte e ao processo de morrer é fundamental para o amplo entendimento do luto" (Oliveira e Silva, 2014, p. 71). Por isso, nos propusemos a refletir sobre as mudanças desse processo na pandemia da Covid-19.

Em dezembro de 2019, foi reportada na China uma doença causada pelo novo coronavírus, a Covid-19 (Ho, Chee, \& Ho, 2020). Com a globalização, sua transmissibilidade permitiu que casos aparecessem 
em diversos países do mundo, com rápido crescimento dos afetados e de mortes. Em março de 2020, a Organização Mundial da Saúde (OMS) qualificou o momento como pandemia (https://bit.ly/2Y4HwWI).

Em curto tempo, observa-se um número elevado de perdas. Além disso, a necessidade de isolamento inviabiliza a participação dos familiares e amigos no cuidado e nos rituais de despedida, afetando a vivência da terminalidade e da morte. Essas novas nuances podem impactar a saúde mental da população, promovendo alterações emocionais, cognitivas e comportamentais (Fundação Oswaldo Cruz, 2020e). A pandemia, portanto, caracteriza-se como um período de crise psicológica (Weir, 2020) e suas implicações para a saúde mental podem se estender por mais tempo que a vigência da doença, conforme evidenciado em tragédias anteriores (Ornell, Schuch, Sordi, \& Kessler, 2020).

A OMS (2007) ressalta a necessidade de planejamento e preparo para o enfrentamento de crises e desastres, com intervenções em três etapas: pré-crítica ou de prevenção; crítica ou de emergência propriamente dita; e pós-crítica, pós-emergências ou de recuperação. Franco $(2012,2015)$ destaca a importância da organização de respostas a tais situações, visando aliviar o sofrimento imediato e minimizar danos, mediante apoio para enfrentamento da crise. No momento, atentamo-nos à segunda e terceira etapas, à medida que estão sendo desenvolvidas, em nosso país, estratégias para lidar com o momento de crise aguda já instalada e ações visando mitigar consequências futuras.

Assim, reforçamos a necessidade de políticas públicas voltadas para saúde mental que visem estabelecer medidas precoces e que previnam agravos posteriores, valorizando "a implementação rápida e estendida das ações de Saúde Mental e Apoio
Psicossocial (SMAPS) para compreender e mapear questões de SMAPS, de forma a prevenir as possíveis repercussões a médio e longo prazo" (Fiocruz, 2020h). Essas ações devem ser voltadas para uma atenção integral, desde ações de base sociocomunitária até os cuidados especializados.

Este ensaio tem como objetivo, portanto, compreender o luto no âmbito da Covid-19, ressaltando a importância da adaptação e da criação de estratégias de assistência, incluindo políticas públicas voltadas para o cuidado integral à saúde mental em processos de enlutamento.

\section{Concepções de uma vivência individual e coletiva}

Desde que nascemos, vivenciamos inúmeros tipos de perda, seja porque alguém morreu, porque estamos crescendo, mudando de cidade, de emprego ou de relacionamento. Todos esses cortes podem mobilizar alterações emocionais, cognitivas e comportamentais, na medida em que o luto responde a perdas reais e simbólicas (Cavalcanti, Samczuk, \& Bonfim, 2013).

Contudo há particularidades na perda por morte, por seu caráter irreversível, com consequente abordagem de cuidados diferenciada (Parkes, 1998). Dessa forma, partiremos da concepção de luto "como reação à perda de um ser, com o qual se possui vínculo afetivo, devido à morte" (Oliveira e Silva, 2014, p. 71).

O luto é entendido como um processo normal e dinâmico, desencadeado pelo rompimento de um vínculo significativo, com impacto em diferentes esferas da vida do sujeito (Bowlby, 1989; Kreuz \& Franco, 2017; Parkes, 1998). Worden (2013) organizou, em quatro categorias, as reações esperadas no processo normal de enlutamento, tais como evidenciadas na Tabela 1.

Tabela 1. Reações ao luto, segundo Worden (2013)

\section{Sentimentos \\ Tristeza, raiva, culpa e autocensura, ansiedade, solidão, fadiga, desamparo, choque, saudade, libertação, alívio e torpor}

\section{Cognições}

Descrença, confusão, preocupação, sensação de presença e alucinações

\begin{abstract}
Sensações físicas
Vazio no estômago, aperto no peito, aperto na garganta, hipersensibilidade a ruídos, sensação de despersonalização, dificuldade em respirar, fraqueza muscular, falta de energia e boca seca.
\end{abstract}

\section{Comportamentos}

Distúrbios de sono, distúrbios de apetite, ausência de pensamento, isolamento social, sonhos com a pessoa que morreu, evitação das lembranças, procurar e chamar pela pessoa, suspiros, hiperatividade e agitação, choro, visita a lugares e/ou carregar objetos que lembrem o falecido, valorização dos objetos do morto.
O processo de luto foi descrito, historicamente, por meio de estágios (Kubler-Ross, 1969/2008), fases (Bowlby, 1989; Parkes, 1998) ou tarefas (Worden, 2013). Contudo essas classificações vêm sendo problematizadas, considerando o caráter singular, e não linear desse momento. Compreende-se atualmente que "não há duração determinada, nem fases preestabelecidas e que, para cada tipo de perda, devemos considerar os fatores e circunstâncias relacionados à perda e seus significados" (Kreuz \& Franco, 2017, p. 170).

Experiências de enlutamento são, assim, individuais e únicas (Alves \& Kovács, 2016), e não sintomas que se 
iniciam com a morte e findam com o tempo (Parkes, 1998). O trabalho com o luto promove "a expressão de emoções sem reservas (por mais inadequadas e contraditórias que elas possam ser), envolve a adaptação à nova noção interna de mundo, assim como ações práticas sobre a nova realidade que se apresenta, agora sem a pessoa amada" (Solano, 2014, p. 109), facilitando a vivência individual da perda de maneira ativa.

O luto foi descrito de maneira menos linear no "Modelo do Processo Dual" (Stroebe \& Schut, 2010) que questiona teorias tradicionais com propostas elaborativas. Aqui, o sujeito enlutado vivencia um processo dinâmico e adaptativo, com enfrentamento a partir da oscilação entre confrontação e evitação dos estressores primários - orientados para perda - e secundários - orientados para restauração (Mazorra, 2009; Stroebe \& Schut, 2010).

Socialmente, fala-se em superação ou finalização do luto. Todavia acredita-se que tais termos não abarcam a riqueza e a complexidade de tal caminhar, pois se trata de uma transição da realidade desadaptada para uma realidade adaptada, sem a presença da pessoa (Kovács, 1992). Entende-se, então, que a elaboração do luto é constante e há uma transformação contínua do vínculo com a pessoa amada, de modo que o vínculo presencial é rompido, mas este existe com as memórias e com a lembrança do ente querido.

Tal como descrito, são esperadas reações para o chamado "luto normal", entendido como processo natural que faz parte da existência humana, na medida em que a dor é consequência do rompimento do vínculo de amor e compromisso (Bowlby, 1989; Parkes, 1998). Entretanto, dependendo da intensidade, da duração e do modo como tais respostas prejudicam a relação do enlutado consigo, com o mundo e com os outros, pode-se indicar a vivência de um luto complicado. Este se trata de uma paralisação em um dos polos descritos no processo dual, em que o sujeito se mantém orientado somente para perda ou para restauração (Stroebe \& Schut, 2010).

Como sinais de adoecimento, identificam-se pensamentos invasivos, recorrentes e persistentes com a pessoa ou com o episódio da morte, tristeza intensa, embotamento afetivo considerável, afastamento e conflitos nas relações interpessoais, falta de sentido na vida e até pensamentos e ideação suicida (Schmidt, Crepaldi, Bolze, Neiva-Silva, \& Demenech, 2020).

São sinais de alerta: mudanças de humor e de comportamento, dificuldade de concentração, agressividade, cansaço, mudança na regulação do sono e da fome, além de problemas somáticos (Campos, 2013).

O DSM-V aponta que depois de doze meses, caso os sintomas sejam persistentes e tragam prejuízos à funcionalidade do sujeito, este passa a ser diagnosticado com o Transtorno do Luto Complexo Persistente (American Psychiatric Association, 2014). Existem controvérsias e críticas relacionadas a essa classificação diagnóstica. Há perspectivas que ressaltam que o diagnóstico para o luto e sua inclusão no manual de psicopatologia permitem que haja maior capacitação dos clínicos para identificar o luto complicado e o encaminhamento para um manejo especializado. Outros trazem críticas quanto à categorização nosológica, indo de encontro à patologização desse processo, pois isso pode normatizar, silenciar e prejudicar a vivência do sofrimento dos enlutados, ressaltando-se a importância de uma avaliação apurada e criteriosa do processo para o devido cuidado e tratamento (Rando et al., 2012).

É crucial considerar que, mais do que a duração ou a presença de comportamentos específicos, o que pode definir o luto como complicado é a intensidade dos sintomas que colocam a vida do enlutado em risco de adoecimento, morte ou suicídio, além da dificuldade em se adaptar à realidade após a perda (Marques, 2015). Ademais, há dificuldade em estabelecer critérios de referência para a duração e a expressão do luto devido às variações nos grupos culturais e processos individuais (Michel \& Freitas, 2019).

Cabe destacar os fatores de proteção e complicadores que influenciam a vivência desse decurso. Aqui, são considerados aspectos relacionais, como tipo de relacionamento e vínculo estabelecido com a pessoa falecida; circunstanciais, como a forma que a perda ocorreu; históricos, como a história de vida do enlutado e sua relação com perdas reais e simbólicas anteriores; de personalidade, como traços de personalidade e funcionamento psíquico; e sociais, que abrangem o mediador social como relevante para o enfrentamento da situação (Marques, 2015).

Os fatores de proteção ao luto complicado são: vivência de um apego seguro que permite maior capacidade de integrar novas informações e ativar mecanismos de resiliência; existência de vínculos sem conflitos com a pessoa que faleceu, evitando ambivalências danosas; percepção de apoio social adequado, necessário e suficiente; realização de rituais; possibilidade de despedida; reconhecimento do luto pela sociedade; permissão para vivência do sofrimento; e capacidade de resiliência (Braz \& Franco, 2017).

Já os fatores de complicação são: apego inseguro, no qual os sujeitos são desorganizados e apresentam padrões comportamentais de fracasso; relação de conflito, pendência ou dependência com a pessoa morta; fase do ciclo vital do enlutado e da pessoa que faleceu; apoio social percebido como inadequado, abusivo e insuficiente; família disfuncional e com padrões comunicacionais comprometidos; morte repentina, violenta, aguda e por suicídio; não localização e visualização do corpo; não realização de rituais de despedida; funcionamento psíquico do enlutado; disfunções psíquicas prévias; e perdas múltiplas e sucessivas (Braz \& Franco, 2017). Destaca-se, ainda, o luto não permitido ou não franqueado como fator complicador, pois se nega a permissão social de sofrer, haja vista que as perdas não podem ser abertamente apresentadas, socialmente validadas ou publicamente mantidas (Casellato, 2013).

Ressalte-se que, ainda que se trate de processo vivido singularmente, o luto não se configura como fenômeno puramente individual. A morte afeta, em primeiro nível, toda uma família, a qual terá que elaborar 
os afetos mobilizados pela perda e efetivar mudanças em seu funcionamento. A família pode ser tanto facilitadora quanto dificultadora do processo (Delalibera, Presa, Coelho, Barbosa, \& Franco, 2015).

Outro aspecto social é ocorrência de lutos coletivos que afetam todo um grupo social. Podem ser considerados inclusos desde indivíduos atingidos por situações de desastre (sobreviventes e familiares de pessoas mortas em enchentes, incêndios ou ataques terroristas) até os atingidos via divulgação midiática que passam a se sentir participantes do acontecido, ainda que não tenham convivido com as pessoas falecidas. Nesses casos, as vivências subjetivas serão atravessadas por elementos coletivos, como a construção de memoriais, a mobilização política e a conexão com o senso de pertencimento e identidade grupal (Franco, 2012). O contato com família, amigos, religião e mesmo a comunicação com desconhecidos que partilham vivências de luto semelhantes podem atuar como fatores protetores, restaurando um senso de amparo e familiaridade (Barros \& Borges, 2018).

Seu potencial de atuar como articulador e catalisador no campo político demonstra o aspecto social e coletivo do luto. A partilha da perda e da dor, bem como a necessidade de advogar pelo valor e dignidade de seus mortos ou mesmo pela visibilidade e reconhecimento legal das perdas e das circunstâncias em que ocorreram, desempenha importante papel em diversos movimentos sociais de grupos marginalizados. Quando o luto partilhado impulsiona ações de ordem política, a participação política também participa em seu processo de elaboração (Milstein, 2017).

Importa, então, considerar tal dimensão social na oferta de assistência e elaboração de políticas públicas. Urge ultrapassar uma concepção apartada e fragmentária entre promoção de saúde e atenção ao luto, na qual discursos sobre a primeira presumem que o luto seja questão específica de equipes especializadas em cuidados paliativos e atenção ao luto, restando pouco espaço para a articulação intersetorial e o fortalecimento de recursos comunitários fundamentais no suporte a tal processo (Kellehear, 2007). Mesmo os avanços no reconhecimento dos cuidados paliativos como questão de saúde pública e o consequente desenvolvimento de políticas voltadas a tal assistência não garantiram o equivalente desenvolvimento de políticas de atenção ao luto, especialmente considerando ações que ultrapassem esses serviços especializados (Rumbold \& Aoun, 2014).

A análise de modelos de atenção ao luto, adotados em diferentes países, ressaltou como desafiante a determinação da população alvo da assistência, a verificação de riscos associados ao luto e a avaliação dos serviços ofertados. Nesse contexto, indica-se a necessidade de considerar os modelos de saúde pública com suas características e seus desafios, e de melhorar a avaliação da necessidade de suporte a enlutados, ampliando o papel da atenção primária à saúde (Breen, Aoun, O’Connor, \& Rumbold, 2014).
Por sua vez, estudos empíricos de base populacional afirmam que tanto a existência de diferentes níveis de risco e necessidade de suporte entre família enlutadas (Aoun et al., 2015) como recursos informais e comunitários são os mais buscados pela população, sendo percebidos como cuidados importantes e satisfatórios (Aoun, Breen, White, Rumbold, \& Kellehear, 2018). Sustentam, assim, que estes sejam reconhecidos e fortalecidos pelas políticas públicas como principal fonte de atenção ao luto em articulação com serviços especializados, que possam oferecer cuidados diferenciados para casos de média e alta complexidade.

Adicionalmente, na assistência psicossocial, em situações de desastres, destaca-se a importância de não restringir o suporte aos momentos iniciais, sendo necessário construir settings e estratégias adaptados a cada situação, considerando aspectos grupais e individuais do luto(Franco, 2012). Um exemplo na realidade brasileira é a Estratégia de Saúde Mental elaborada em decorrência do incêndio na Boate Kiss (Noal et al., 2016), que teve como importante demanda a atenção ao luto. A ação envolveu a articulação entre poder público, sociedade civil e organizações internacionais, sendo identificáveis diferentes momentos no planejamento e oferta de assistência, como também a importância da intersetorialidade para garantir cuidado integral, com base na atenção primária e no acesso organizado a diferentes níveis de atenção.

Nessa lógica de organização, a base da assistência estaria situada nas ações de cunho social e comunitário, e, na sequência, na atenção por profissionais generalistas, ficando a assistência especializada em saúde mental já como nível de maior complexidade e destinada a casos de luto cuja resolutividade não possa ser alcançada nos níveis anteriores (Aoun et al., 2015, 2018; Breen et al., 2014; Inter-Agency Standing Committe, 2020a).

As intervenções especializadas podem ser o aconselhamento e a terapia do luto (Worden, 2018), que visam reconhecer e tornar acessíveis melhores ferramentas para auxiliar o sujeito em sua travessia. Estas, embora contribuam para mitigar as reações complicadas do enlutamento, não objetivam sua supressão.

\section{O luto na Covid-19}

A pandemia acarretou inúmeras perdas. Além das mortes em si, há sensação de insegurança e medo constantes, mudanças nas relações sociais e ansiedade em relação ao futuro, à saúde e à estabilidade econômica e social (Miyazaki \& Teodoro, 2020). Dessa forma, a experiência do luto diante dessa crise não afeta todos da mesma forma. É possível falarmos de, pelo menos, três níveis de afetação (Zhai \& Du, 2020).

Há a vivência de um luto primário quando nos referimos às pessoas infectadas, aos familiares destas e aos profissionais de saúde que atuam na linha de frente do combate à pandemia. Compreende-se que estes vivenciam o luto de forma mais direta devido ao contato mais próximo com a perda. A esfera secundária diz respeito 
ao luto simbólico do isolamento e das perdas consecutivas - financeiras, sociais, relacionais e da estabilidade da vida presumida - que as medidas de contenção da pandemia causaram em toda a população (Zhai \& Du, 2020), incluindo o medo constante de contaminação (Schmidt et al., 2020). O luto a nível terciário compete à ansiedade diante da morte à qual todos estão expostos. A pandemia modificou o tabu em torno da morte, pondo a maioria diante da impossibilidade da eternidade, e irrompeu um golpe em nosso narcisismo: não somos tão senhores da nossa vida como acreditamos ser. Tal realidade provocou o encontro, indesejado e estupefato, com a morte.

Podemos dizer que o coronavírus tornou-se um memento mori - "lembre-se da morte" -, expressão usada para ocasiões em que a finitude é lembrada, sendo inserida em elementos simbólicos de obras artísticas como caveiras e foices. Esse lembrete pode ser um convite diário para constatação, aceitação e elaboração da nossa mortalidade como condição existencial. Apesar desse descortinar, há ainda uma evitação em torno do tema e que cada pessoa lidará com esse risco de forma subjetiva, podendo ou não transformar sua relação com a finitude a partir de então.

Há, assim, a necessidade de cuidar de formas variadas de cada um dos níveis de afetação por suas repercussões específicas. Devido à complexidade e à densidade de cada um, e buscando consonância com objetivo deste escrito, focar-se-á no primeiro nível discutido - o luto para pacientes, familiares e equipe de saúde.

Como medida de prevenção da propagação do vírus, os cuidados com paciente no ambiente hospitalar são realizados seguindo protocolos de isolamento. Pessoas hospitalizadas têm contato restrito com a equipe, devidamente paramentada com os equipamentos de proteção individual (EPIs), e com a família, que não tem permissão de visitar ou permanecer no acompanhamento diário, mantendo contato apenas, quando possível, por meio de tecnologias da informação e comunicação (TICs) (Fundação Oswaldo Cruz, 2020e; Schmidt et al., 2020).

Pacientes internados apresentam-se ansiosos e angustiados devido ao severo desconforto respiratório e ao medo do quadro agravar e morrerem sozinhos longe de suas famílias (Schmidt et al., 2020). Há a possibilidade da vivência do luto antecipatório, sendo este uma reação de pesar em pessoas que não estão enlutadas pela morte em si, mas pela experiência com as perdas ocasionadas pela doença, pela separação e distanciamento dos familiares e amigos e pela possibilidade da morte(Lindemann, 1944). A internação em unidades de isolamento e a impossibilidade ou possibilidade de comunicação limitada com a família, de certa forma, iniciam a preparação para trabalhar a possibilidade real de morte.

Esses sujeitos já se encontravam mobilizados emocionalmente pelas condições vivenciadas, seja por eles mesmos, seja empaticamente pelos demais, tendo em vista que a pandemia traz implicações para toda sociedade (Weir, 2020). Contudo a vivência direta de todas essas mudanças pode trazer implicações para saúde mental dos sobreviventes, tanto a curto quanto a longo prazo (Ornell et al., 2020), aumentando o risco para o luto complicado. Além do sofrimento do paciente, ter um ente querido doente e internado, e ainda com o diagnóstico de doença grave de evolução rápida e alto risco, pode mobilizar alterações emocionais na família e modificar sua dinâmica de funcionamento (Delalibera et al., 2015).

Um fator que merece destaque é a restrição de comunicação que pode afetar o processo de luto dos pacientes e familiares. $\mathrm{O}$ paciente, que antes poderia expressar necessidades finais e realizar despedidas essenciais, pode não conseguir elaborar o processo vivenciado por estar experienciando o morrer de maneira solitária, muitas vezes sem atender às necessidades básicas para sua partida. A família, que antes poderia participar dos cuidados, mesmo por meio de visitas ou diálogos com equipe, hoje encontra-se mais ausente, fator que inviabiliza a construção de narrativas do processo de morrer, essencial para elaboração da despedida.

Outro aspecto é ausência ou mudança nos rituais de despedida na iminência do morrer. Falamos do processo entre paciente e familiares antes do morrer, podendo conter ações como incentivo à comunicação, resolução de questões, momentos de agradecimento e perdão, compartilhamento de vivências boas, entre outras capazes de promover momentos de qualidade ao doente e de conforto aos familiares (Lisbôa \& Crepaldi, 2003; Schmidt, Gabarra, \& Gonçalves, 2011; Schmidt et al., 2020). Tais despedidas possibilitam fechamentos, expressão de sentimentos e resolução de pendências que podem atenuar o sofrimento após a perda e assentir ao enlutado a vivência do processo dual de perda e reparação, abrindo caminho para a construção de novos significados.

Após a morte, os rituais funerários apresentam-se como fundamentais para despedida, constatação e elaboração da perda. Rituais de despedida são considerados essenciais à elaboração do luto (Fundação Oswaldo Cruz, 2020e) devido ao seu valor simbólico e social. Eles materializam a perda e, de certa forma, também a negam, apontando para uma explicação espiritual, possibilitando conforto aos que ficam.

Com a Covid-19, velórios e enterros têm sido evitados ou realizados com restrições por orientação de segurança em decorrência do intenso contato que pode ocorrer entre as pessoas. Também não se recomenda a realização da tanatopraxia - limpeza e preparo do corpo - elemento significativo em nossa cultura (Moore, Tulloch, \& Ripoll, 2020; Schmidt et al., 2020). O caixão deve ser lacrado (Ministério da Saúde, 2020), impedindo a família de visualizar o falecido, e as práticas religiosas, que permitem que família e amigos permaneçam perto do corpo para despedida, também são evitadas. Tudo isso pode provocar sentimentos de negligência e desumanização, bem como de injustiça e abandono, aumentando os riscos de adoecimento mental após a crise (Fundação Oswaldo Cruz, 2020e; Franco, 2012; Miyazaki \& Teodoro, 2020) e acarretando implicações 
importantes no processo de enlutamento, por impedirem momentos de homenagem e impossibilitarem - de forma presencial - a construção de um espaço de conforto e apoio psicossocial (Ingravallo, 2020; Schmidt et al., 2020; Wallace, Wladkowski, Gibson, \& White, 2020).

Além disso, a ocorrência de casos de contaminação e morte de membros da mesma família em um curto espaço de tempo traz grande desafio (Schmidt et al., 2020), mobilizando lutos sequenciais e dificultando o processo de despedidas e adaptação, já que o sujeito precisa se reorganizar diante da ausência de mais de uma pessoa com vínculo significativo (Wallace et al., 2020). A rede de apoio sociofamiliar, que seria fator protetor, pode não estar fortalecida, pois seus membros individualmente não conseguem dar conta de se apoiar, devido à sua intensa dor. Em alguns casos, morre-se toda a família, restando apenas um membro. Tal situação ocorre também em desastres aéreos, inundações, entre outros, e esfacela a rede de apoio do enlutado.

Outro fator complicador para o luto é a faixa etária da pessoa falecida. Ainda que os óbitos infantis sejam em menor número no contexto pandêmico atual (Morand et al.,2020), não podemos esquecer que a morte de crianças e adolescentes implica em consequências sociais mais assoladoras. É interpretada como interrupção do ciclo natural da vida, como algo inesperado e trágico, incompatível com a visão de alegria atribuída a essas faixas etárias (Lima, 2019). Por outro lado, percebe-se certo descaso e descuido com a população idosa, considerada grupo de risco, sendo o óbito nessa faixa etária mais socialmente aceito. No contexto da Covid-19, que afeta, em sua grande maioria, a população idosa, há algumas elucubrações bárbaras que consideram a vida do idoso uma vida residual ou uma morte esperada. Não são raras as manifestações públicas nas redes sociais ou em pronunciamentos na televisão que banalizam tais mortes, invalidando o sofrimento decorrente destas (https://bit.ly/2WBaRYG).

Finalmente, ressaltamos a atenção especial que deve ser voltada à equipe de saúde e sua experiência de luto na crise da Covid-19 devido à intensidade de vivências desta em torno da morte pelo elevado número de perdas, agravado pelo modo como historicamente os profissionais de saúde são preparados para lidar com a finitude. De modo geral, o trabalhador de saúde é distanciado do seu objeto de intervenção e executa práticas repetitivas, protocoladas, sem reflexão crítica e envolvimento afetivo, tomando a morte como inimiga que deve ser combatida a qualquer custo. $\mathrm{O}$ distanciamento dos profissionais para encarar o óbito e a finitude prejudica o cuidado eficaz a si mesmos, ao paciente e a seus familiares (Lima \& Andrade, 2017).

Entretanto, no caso da Covid-19, percebe-se uma dificuldade maior, não com o falecimento em si, mas com esse tipo e as especificidades da atuação nesse contexto: muitas horas de trabalho; uso de EPIs próprios; poucas condições para uma atuação segura e digna; horas sem poder tirar os EPIs; sem poder ir ao banheiro ou beber água; pouco ou nenhum momento para descanso; medo constante de se infectar e de infectar seus entes queridos; além do medo de morrer, agora não só na fantasia, mas também na realidade. Adicionam-se a isso profissionais adoecendo por sobrecarga e Burnout, com risco inclusive de sofrer de estresse pós-traumático.

Ao ser posto no lugar de herói invencível - lema constante para se referir aos profissionais da linha de frente do combate ao Covid-19 -, o profissional tem negada a permissão social de sofrer, e ocorre o que chamamos de luto não autorizado, podendo dificultar a elaboração das perdas pela ausência de aprovação social do seu padecimento (Braz \& Franco, 2017).

Após termos detalhado as particularidades de cada grupo, precisamos destacar aspectos fundamentais de afetação à tríade. A banalização da morte e a ausência ou mudança na forma de suporte social para o enfrentamento do sofrimento são fatores de influência importantes para lutos complicados. O número alarmante de mortos e sua exploração incessante na mídia irrompem o fenômeno da morte escancarada e sua banalização, podendo contribuir para quadros patológicos e traumáticos em torno da perda (Campos, 2013). Cenários de não valorização da dor do outro e não permissão para que os enlutados vivenciem sua perda de forma presencial - ou nos modelos conhecidos até então - são fatores de alerta para lutos complicados, pois dificultam que a pessoa viva seu processo de adaptação (Braz \& Franco, 2017).

O apoio social é fundamental para auxiliar o enlutado (Miyazaki \& Teodoro, 2020), sendo imprescindível a criação de espaços para expressão e reconhecimento dos sentimentos, pois, ao transformar a separação em reparação, ele pode retomar o fluxo da vida (Campos, 2013). A possibilidade de estar com o outro em seu sofrimento, deixando que se expresse e produza significados para sua dor, é fundamental para evitar a irrupção de traumas psíquicos ou prevenir agravos mais severos (Lima, 2019).

É crucial que se perceba também um importante fator organizador do luto: o desenvolvimento e a manifestação da resiliência, considerada como a capacidade de criar alternativas flexíveis e criativas para a saída da crise (Braz \& Franco, 2017). Assim, alerta-se que, por conta de todos os fatores citados, é possível que se produzam lutos complicados e sofrimentos psíquicos intensos, mas que isso não se dá a priori e sem que diversas inter-relações e tramas sejam estabelecidas. Portanto é relevante considerar as capacidades de reorganização e simbolização de cada sujeito e a existência de suporte social e afetivo - mesmo que virtual - e estratégias de cuidado em saúde mental.

\section{(Re)Pensando estratégias de cuidado ao luto na Covid-19}

Retomando elementos abordados, ressalta-se a importância de pensar estratégias de atenção psicossocial 
em resposta ao cuidado com o luto na pandemia. Diante da inexistência de uma política nacional de atenção ao luto, este momento terá o desafio de fazer avançar articulações entre diferentes setores de cuidado, podendo colocar-se como catalisador de avanços duradouros na atenção ao luto em saúde pública.

Cabe destacar a confluência entre indicações apresentadas para políticas voltadas ao luto (Aoun et al., 2015, 2018; Breen et al., 2014) e recomendações para a atenção psicossocial na pandemia (Inter-Agency Standing Committe, 2020a), ambas apontando para o fortalecimento de ações de base sociocomunitária, com reconhecimento de diferentes níveis de complexidade das demandas e acesso organizado à atenção especializada. É também imprescindível diferenciar os momentos de cuidado, reconhecendo especificidades das etapas de preparação e planejamento, atenção imediata no contexto de fim de vida e suporte ao luto em médio e longo prazos (Franco, 2015; World Health Organization, 2007). Serão apresentadas, então, possibilidades voltadas a diferentes momentos e níveis de complexidade.

Diante do contexto emergencial da pandemia, foram produzidas cartilhas virtuais por ONGs, associações profissionais e órgãos de vigilância sanitária. Esses documentos oferecem informações envolvendo a doença, seus desdobramentos e novas ações de cuidado que precisam ser incorporadas à rotina da população, apresentando-se como possibilidades de ação de educação em saúde adaptadas ao contexto de distanciamento social. Algumas, ainda que não abordem o luto diretamente, trazem a ideia de finitude e a possibilidade de que a doença conduza alguém próximo à morte. Outras são direcionadas a promover novas estratégias de cuidado àqueles em fase de terminalidade pela Covid-19 e aos seus familiares, conectando-se tanto com o contexto do luto antecipatório quanto com a elaboração do luto após o óbito (Tabela 2).

Em conformidade com as cartilhas sobre a assistência ao luto na pandemia, pode-se conversar sobre possibilidades de se criar memoriais em homenagem às vítimas do coronavírus (Almeida, 2020; Fundação Oswaldo Cruz, 2020e; Wang, Teo, Yee, \& Chai, 2020). A ideia não é focar na imagem da catástrofe por si, mas evocar elementos provenientes das narrativas daqueles que vivenciaram a tragédia. A morte pode representar o fim da vida dos que se foram, mas o marco da ausência permanece para aqueles que vivenciam o luto. Reitera-se que os desastres, a partir da metade do século XX, passam a ter caráter de luto público, sendo os memoriais seus representantes concretos (Walter, 2008).

Tabela 2. Cartilhas que abordam o luto no contexto da Covid-19

$\begin{array}{lll}\text { Objetivo Título do documento } & \text { Autor/órgão }\end{array}$

Cartilha para enfrentamento do estresse em tempos de pandemia Weide et al. (2020)

A história da ostra e da borboleta: o coronavírus e eu

Gomez (2020)

Meu Herói és tu: como as crianças podem combater o Covid-19

Inter-Agency Standing Committee (2020)

Conexão indireta com o luto:

abordam a finitude e a possibilidade Saúde mental e atenção psicossocial na pandemia Covid-19

de que a doença conduza à morte

Fundação Oswaldo

Cruz (2020i)

Fundação Oswaldo

Cruz (2020g)

Fundação Oswaldo

Cruz (2020d)

Covid-19: povos indigenas no contexto da Covid-19

Saúde mental e atenção psicossocial na pandemia

Covid-19: Covid-19 e população privada de liberdade
Fundação Oswaldo

Cruz (2020b) 
Tabela 2. Continuação

$\begin{array}{lll}\text { Objetivo } & \text { Título do documento } & \text { Autor/órgão }\end{array}$

"Novos rituais do luto em tempos de distanciamento"

Almeida (2020)

Como comunicar às crianças a morte de um ente querido por Covid-19

Lima e Lopes (2020)

\section{Conexão direta com a temática do luto:}

sugerem novas estratégias de cuidado àqueles em fase de terminalidade pela Covid-19 ou aos seus familiares, inclusive após o óbito do paciente
Saúde mental e atenção psicossocial na pandemia

Covid-19: orientação aos psicólogos hospitalares

Saúde mental e atenção psicossocial na pandemia

Covid-19: suicídio na pandemia Covid-19

Saúde mental e atenção psicossocial na pandemia

Covid-19: recomendação para gestores

Saúde mental e atenção psicossocial na pandemia

Covid-19: processo de luto no contexto da Covid-19

Cuidados paliativos: orientações aos profissionais de saúde
Fundação Oswaldo

Cruz (2020c)

Fundação Oswaldo

Cruz (2020j)

Fundação Oswaldo

Cruz (2020f)

Fundação Oswaldo

Cruz (2020e)

Fundação Oswaldo

Cruz (2020a)
Memoriais arquitetônicos são tradicionais na história da humanidade, construídos para homenagear vítimas de guerra ou ataques, a exemplo dos de Washington, Hiroshima e Nagasaki (Silva, 2012), patrimonializando a dor das perdas em massa. Na cultura brasileira, a preparação para estratégias de enfrentamento aos danos psicossociais ocasionados por desastres é ainda incipiente, incluindo a realização de rituais de despedida e de elaboração do luto coletivo, tais como a construção de memoriais.

No Brasil, foram criados memoriais virtuais em homenagem às vítimas da Covid-19, como Inumeráveis (https://bit.ly/2WE05AL) e Santinhos (https://bit.ly/3ix4a1s). Qualquer pessoa pode enviar nome, idade e uma história que represente o falecido, como forma de reiterar que as vidas não se reduzem à estatística. O relicário virtual "Reliquia.rum" (https://bit. ly/3oBwOCn), por sua vez, conta a história de mulheres brasileiras vítimas da Covid-19.

$\mathrm{Na}$ era digital, tem se ampliado o debate em torno da preservação de perfis de falecidos nas redes sociais como possibilidade de interação e atenuação da dor da morte, fortalecendo, assim, a publicização da dor do luto (Ramos, 2015). O Facebook, por exemplo, fornece a possibilidade de o usuário escolher se deseja que sua conta seja transformada em memorial ou se deseja excluí-la da rede. Ao longo do processo de morrer e no pós-morte, o fortalecimento das redes religiosas e/ou espirituais preexistentes do falecido e dos enlutados é de fundamental importância (Sbizera, 2018) por mitigar posteriores complicações ao processo de luto. Podem ser incentivados contatos virtuais com líderes religiosos importantes para a família e que sejam significativos para esse momento (Fundação Oswaldo Cruz, 2020e). Em muitos países, rituais fúnebres têm acontecido com transmissão ao vivo ou gravados para serem transmitidos posteriormente.

Embora esses recursos não substituam os rituais funerários tradicionais, é possível que auxiliem pessoas a se despedir dos falecidos e a se apoiar mutuamente, ainda que virtualmente, repercutindo na dimensão social da morte e do morrer (Ingravallo, 2020). Não obstante, nem todos se beneficiam desses recursos, tanto pela falta de acesso quanto pelas dificuldades para utilizar os equipamentos necessários, o que costuma ocorrer particularmente entre idosos (Schmidt et al., 2020).

Os lutos expressos em memoriais virtuais apresentam semelhanças entre modos de enlutar atuais e medievais no tocante ao compartilhamento com a coletividade, mediante a expressão do pesar, dos afetos e dos pensamentos ligados a ele. Nesse sentido, ressalta-se que há uma pressão social para a manifestação desses lutos, abrindo espaço para o julgamento do outro na interatividade proporcionada por plataformas virtuais (Walter, 2015). 
No contexto dos cuidados em fim de vida, o trabalho com equipes deve ocorrer no sentido de promoção de qualidade de vida e estabelecimento de estratégias de enfrentamento adaptativas por meio de psicoeducação, acolhimento e escuta empática. Propõe-se possibilitar a construção de sentido para as perdas vivenciadas, resgatando a humanidade presente em cada profissional e, como consequência, reumanizando sua prática (Schmidt et al., 2020).

Recentes estudos (Cusso, Navarro, Gálvez, 2020; Ingravallo, 2020; Wang et al., 2020) discutem a humanização no suporte de pacientes e familiares que atravessam a terminalidade da Covid-19. São propostas possibilidades de despedida entre familiares e pacientes, e de acompanhamento dos enlutados nas homenagens póstumas. $\mathrm{O}$ trabalho com os pacientes dependerá da sua condição clínica, pois é bastante comum estarem intubados e sem possibilidade de comunicação.

Indica-se que, quando possível, o paciente tenha contato virtual com sua família e que esta seja informada diariamente acerca do estado de saúde dele, sendo acionada quando ocorrer alguma mudança significativa. Recomenda-se que a equipe de saúde faça a mediação de uma videoconferência entre o paciente e seus entes queridos, permitindo a dignificação do processo de morrer e a realização de despedidas e fechamentos que amenizam o impacto de adoecimentos mentais nos familiares (Fundação Oswaldo Cruz, 2020c; Grincenkov, 2020; Wang et al., 2020). Considerando aspectos éticos e legais, sugere-se que, na admissão, o cuidador principal assine um Termo de Responsabilidade pelo uso e divulgação de imagens e informações do paciente. Deve-se proporcionar, dentro das possibilidades, o uso de recursos como: telefonemas; mensagens de texto, áudio e vídeo; redes sociais; cartas; desenhos; recados; entrega de objetos significativos; entre outros, devendo ocorrer também o trabalho com os pensamentos e sentimentos decorrentes da experiência (Fundação Oswaldo Cruz, 2020e; Grincenkov, 2020; Shojaei \& Masoumi, 2020; Wang et al., 2020).

Na comunicação de notícias difíceis, sugere-se que o médico informe o óbito,já que o psicólogo não tem capacitação para transmitir informações sobre o processo de cuidado e da causa da morte. Entretanto é importante que o psicólogo esteja presente para acolher os aspectos emocionais manifestados, podendo oferecer acompanhamento aos enlutados por telefone ou vídeo, subsequente ao funeral, considerando-se o vínculo já estabelecido (Conselho Federal de Psicologia, 2020a; Fundação Oswaldo Cruz; 2020c).

Mediante a assistência em cuidados paliativos do paciente em situação crítica, podem-se identificar os lutos com potenciais de complicação e realizar encaminhamentos para terapêuticas específicas (Wang et al., 2020). Todavia haverá enlutados que não necessitarão desse acompanhamento especializado, podendo ser encaminhados para rede de apoio territorial, preservada a possibilidade de identificação de complicações posteriores na elaboração do luto, com encaminhamento aos serviços adequados.
Assim, reconhecemos a relevância de intervenções psicológicas tanto durante quanto após a vigência da pandemia, considerando também a chamada "segunda onda" (Shojaei \& Masoumi, 2020), com destaque às demandas relacionadas aos processos de terminalidade, morte e luto (Taylor, 2019; Weir, 2020).

A atenção psicológica emergencial no luto pode ter tempo limitado (Worden, 2018), e estratégias remotas podem subsidiar essa terapêutica mediante uso de TICs, de modo síncrono ou assíncrono. Não sendo o foco presente, cabe destacar que, a fim de evitar o alastramento da pandemia, o sistema Conselhos de Psicologia flexibilizou os serviços prestados por meio de TICs. A Resolução $\mathrm{n}^{\circ} 4$, de 26 de março de 2020, simplifica o cadastro obrigatório de psicólogos na plataforma E-Psi para atendimentos remotos, a partir do mês de março de 2020 , suspendendo a vedação anteriormente vigente de atendimentos remotos de urgência e emergência, como a psicoterapia para enlutados no contexto de Emergências e Desastres (Conselho Federal de Psicologia, 2020b).

Considerando o exposto, reiteramos a urgência de pensarmos na articulação de ações, desde o nível de atenção comunitária até níveis de atenção mais especializados, visando ao suporte ao luto individual e ao desenvolvimento de novos modos de reconstrução das perdas em massa, desencadeadas pela pandemia. Cabe destacar a importância do desenvolvimento de uma política interdisciplinar de posvenção, seja remota, seja presencial, quando possível. Entendemos que essas estratégias psicossociais de enfrentamento estão intimamente relacionadas ao processo de luto coletivo e, por isso, devem ser refletidas intersetorialmente, no sentido de elaborar as perdas e prevenir maiores agravos à saúde mental.

\section{Considerações finais}

O processo de luto sempre foi assunto complexo, posto que é envolto por diversos aspectos, com descrições gerais e vivências singulares. A constatação é que a pandemia trouxe mudanças na forma como os sujeitos e a coletividade experienciam o morrer, o que qualifica a particularidade ainda maior desse processo. Dessa maneira, o momento convoca a repensar conceitos e (re)criar compreensões acerca da morte na sociedade contemporânea e do luto diante de tantas mudanças.

Evidenciamos inúmeras iniciativas, de instituições de referência ou profissionais autônomos, na tentativa de mitigar os efeitos emocionais, cognitivos e comportamentais na população geral ou, mais precisamente, naqueles que têm maior risco de complicações, a saber: pacientes, familiares e equipe de saúde da linha de frente. Contudo ressaltamos a importância do fortalecimento da Rede de Saúde Mental para o cuidado com um momento tão único e peculiar. 


\section{The pain that cannot remain silent: reflections on grief in Covid-19 times}

Abstract: Covid-19 caused a high number of losses and changes in the end-of-life care and farewell processes, factors that can add difficulty to the mourning process. Thus, we aim to understand the particularities of grief within the scope of Covid-19, emphasizing the importance of adaptation and creation of strategies to address mental health care in mourning processes. The essay provides a theoretical overview, indicating elements present in the pandemic with the potential to interfere in mourning and presents a survey of initiatives adapted to this context. The pandemic brought changes in the way of experiencing dying. Attempts to mitigate emotional, cognitive and behavioral effects are identified. The moment calls for rethinking concepts and (re) creating understandings about death and grief, emphasizing the importance of a mental health network, with the strengthening of community based actions, recognition of different levels of complexity of demands and organized access to specialized attention.

Keywords: bereavement, grief, pandemics, psychosocial impact, coronavirus infections.

\section{La douleur qui ne peut pas rester silencieuse: réflexions sur le deuil dans le cadre de Covid-19}

Résumé: Le Covid-19 a causé un grand nombre de pertes et de changements dans les processus de soin et d'adieu, facteurs qui peuvent rendre le deuil plus compliqué. Ainsi, nous visons à comprendre les particularités du deuil dans le cadre de Covid-19, en soulignant l'importance de l'adaptation et de la création de stratégies pour les soins dans le domaine de la santé mentale dans les processus de deuil. L'essai fournit un aperçu théorique, indiquant les éléments présents dans la pandémie susceptibles d'interférer dans le deuil et présente des initiatives adaptées à ce contexte. La pandémie a entraîné des changements dans la appréhension la mort. Des initiatives visant à atténuer les effets émotionnels, cognitifs et comportementaux sont mis en évidence. Le moment appelle à repenser les concepts et à (re)créer des compréhensions sur la mort et le deuil, en soulignant l'importance du réseau de santé mentale, avec le renforcement des actions sociocommunautaires, la reconnaissance des différents niveaux de complexité des demandes et l'accès organisé à l'attention spécialisée.

Mots-clés: deuil, douleur, pandémies, impact psychosocial, infections à coronavirus.

\section{Dolor que no puede permanecer en silencio: reflexiones sobre el duelo en tiempos de Covid-19}

Resumen: Covid-19 causó una gran cantidad de muertes y cambios en los procesos de atención y despedida, factores que pueden dificultar el duelo. Por lo tanto, nuestro objetivo es comprender las particularidades del duelo el contexto de Covid-19, enfatizando la importancia de la adaptación y de la creación de estrategias dirigidas a la atención a la salud mental en los procesos de duelo. El ensayo proporciona un panorama teórico, indicando los elementos presentes en la pandemia con potencial de interferir en el duelo y presenta iniciativas adaptadas a este contexto. La pandemia trajo cambios en la forma de experimentar la muerte. Iniciativas que objetivan mitigar los efectos emocionales, cognitivos y conductuales son evidenciadas. El momento requiere repensar conceptos y crear comprensiones sobre la muerte y el duelo, enfatizando la importancia de una red de salud mental, con fortalecimiento de acciones basadas en la comunidad, reconocimiento de diferentes niveles de complejidad de las demandas y el acceso organizado a la atención especializada.

Palabras clave: aflicción, duelo, pandemias, impacto psicosocial, infecciones por coronavirus.

\section{Referências}

Almeida, C. (2020). Novos rituais do luto em tempos de distanciamento. Vamos falar sobre o luto? Recuperado de https://bit.ly/3oiEnxG

Alves, E. G. R., \& Kovács, M. J. (2016). Morte de aluno: luto na escola. Psicologia Escolar e Educacional, 20(2), 403-406. doi: 10.1590/2175-353920150202990

American Psychiatric Association. (2014). Manual diagnóstico e estatístico de transtornos mentais: DSM-5 (5a ed). Porto Alegre, RS: Artmed.
Aoun, S. M., Breen, L. J., Howting, D. A., Rumbold, B., McNamara, B., \& Hegney, D. (2015). Who needs bereavement support? A population based survey of bereavement risk and support need. PloS One, 10(3), 1-14. Recuperado de https://bit.ly/39MZcJ8

Aoun, S. M., Breen, L. J., White, I., Rumbold, B., \& Kellehear, A. (2018). What sources of bereavement support are perceived helpful by bereaved people and why? Empirical evidence for the compassionate communities approach. 
Palliative Medicine, 32(8), 1378-1388. Recuperado de https://bit.ly/3CSeusy

Ariès, P. (2003). História da morte no ocidente: da idade média aos nossos dias. Rio de Janeiro, RJ: Ediouro. (Trabalho original publicado em 1977)

Barros, A. F. O., \& Borges, L. M. (2018). Reconstrução em movimento: impactos do terremoto de 2010 em imigrantes haitianos. Psicologia: Ciência e Profissão, 38(1), 157-171. doi: 10.1590/1982-3703003122016

Bowlby, J. (1989). Uma base segura: aplicações clínicas da teoria do apego. Porto Alegre, RS: Artes Médicas.

Braz, M. S., \& Franco, M. H. P. (2017). Profissionais paliativistas e suas contribuições na prevenção de luto complicado. Psicologia: Ciência e Profissão, 37(1), 90-105. doi: 10.1590/1982-3703001702016

Breen, L. J., Aoun, S. M., O’Connor, M., \& Rumbold, B. (2014). Bridging the gaps in palliative care bereavement support: an international perspective. Death Studies, 38(1), 54-61.

Campos, M. T. F. S. (2013). A influência do luto no comportamento alimentare suas implicações nas condutas nutricionais. Revista Ciência \& Saúde Coletiva, 18(9), 2769-2779. doi: 10.1590/S1413-81232013000900032

Casellato, G. (2013). Dor silenciosa ou dor silenciada? Perdas e lutos não reconhecidos por enlutados $e$ sociedade. São Paulo, SP: Pollo Books.

Cavalcanti, A. K. S., Samczuk, M. L., \& Bonfim, T. E. (2013). O conceito psicanalítico do luto: uma perspectiva a partir de Freud e Klein. Psicólogo InFormação, 17(17), 87-105. Recuperado de https://bit.ly/3D3aNAC

Conselho Federal de Psicologia. (2020a). Oficio-Circular $n^{o}$ 65/2020/GTec/CG-CFP. Recomendações sobre comunicação de óbito por psicólogas. Recuperado de https://bit.ly/3F432vP

Conselho Federal de Psicologia. (2020b). Resolução CFP n ${ }^{\circ} 4$, de 26 e março de 2020. Dispõe sobre regulamentação de serviços psicológicos prestados por meio de tecnologia da informação e da comunicação durante a pandemia do Covid-19. Recuperado de https://bit.ly/3utCKhW

Cusso, R. A., Navarro, C. N., Gálvez, \& A. M. P. (2020). El cuidado humanizado en la muerte por Covid-19: a propósito de un caso. Enfermería Clínica, 31, S62-S67.

Delalibera, M., Presa, J., Coelho, A., Barbosa, A., \& Franco, M. H. P. (2015). A dinâmica familiar no processo de luto: revisão sistemática da literatura. Ciência \& Saúde Coletiva, 20(4), 1119-1134. doi: 10.1590/141381232015204.09562014

Franco, M. H. P. (2012). Crises e desastres: a resposta psicológica diante do luto. O Mundo da Saúde, 36(1), 54-58. Recuperado de https://bit.ly/3kVAEUP

Franco, M. H. P. (Org.). (2015). A intervenção psicológica em emergências: fundamentos para a prática. São Paulo, SP: Summus.

Fundação Oswaldo Cruz. (2020a). Cuidados paliativos: orientações aos profissionais de saúde. Rio de Janeiro, RJ: Fiocruz.

Fundação Oswaldo Cruz. (2020b). Saúde mental e atenção psicossocial na pandemia Covid-19: Covid-19 e população privada de liberdade. Rio de Janeiro, RJ: Fiocruz.

Fundação Oswaldo Cruz. (2020c). Saúde mental e atenção psicossocial na pandemia Covid-19: orientação aos psicólogos hospitalares. Rio de Janeiro, RJ: Fiocruz.

Fundação Oswaldo Cruz. (2020d). Saúde mental e atenção psicossocial na pandemia Covid-19: povos indígenas no contexto da Covid-19. Rio de Janeiro, RJ: Fiocruz.

Fundação Oswaldo Cruz. (2020e). Saúde mental e atenção psicossocial na pandemia Covid-19: processo de luto no contexto da Covid-19. Rio de Janeiro, RJ: Fiocruz.

Fundação Oswaldo Cruz. (2020f). Saúde mental e atenção psicossocial na pandemia: recomendação para gestores. Rio de Janeiro, RJ: Fiocruz.

Fundação OswaldoCruz.(2020g). Saúdementaleatençãopsicossocial na pandemia Covid-19: recomendações aos trabalhadores $e$ cuidadores de idosos. Rio de Janeiro, RJ: Fiocruz.

Fundação Oswaldo Cruz. (2020h). Saúde mental e atenção psicossocial na pandemia Covid-19: recomendações gerais. Rio de Janeiro, RJ: Fiocruz.

Fundação Oswaldo Cruz. (2020i). Saúde mental e atenção psicossocial na pandemia Covid-19. Rio de Janeiro, RJ: Fiocruz.

Fundação Oswaldo Cruz. (2020j). Saúde mental e atenção psicossocial na pandemia Covid-19: suicídio na pandemia Covid-19. Rio de Janeiro, RJ: Fiocruz.

Gomez, A. (2020). A história da ostra e da borboleta: o coronavirus e eu. Recuperado de https://bit.ly/3F2oEsC

Grincenkov, F. R. S. (2020). A psicologia hospitalar e da saúde no enfrentamento do coronavírus: necessidade e proposta de atuação. HU Revista, 45(1), 13-21. Recuperado de https://bit.ly/3uoy90k

Ho, C. S., Chee, C. Y., \& Ho, R. C. (2020). Mental health strategies to combat the psychological impact of Covid-19 beyond paranoia and panic. Annals of the Academy of Medicine Forthcoming, 49(1), 1-3. Recuperado de https://bit.ly/2Y0Og88

Ingravallo, F. (2020). Death in the era of the Covid-19 pandemic. The Lancet Public Health, 5(5), e258. Recuperado de https://bit.ly/2YhR51D

Inter-Agency Standing Committe. (2020a). Como lidar com os aspectos psicossociais e de saúde mental referentes ao surto de Covid-19 (Versão 1.5). Genebra: Iasc.

Inter-Agency Standing Committee. (2020b). Meu herói és tu: como as crianças podem combater o Covid-19. Genebra: Iasc.

Kellehear, A. (2007). The end of death in late modernity: an emerging public health challenge. Critical Public Health, 17(1), 71-79.

Kovács, M. J. (1992). Morte e desenvolvimento humano. São Paulo, SP: Caso do Psicólogo.

Kovács, M. J. (2003). Educação para a morte: temas e reflexões. São Paulo, SP: Casa do Psicólogo.

Kovács, M. J. (2012). Educadores ea morte. Revista Semestral da Associação Brasileira de Psicologia Escolar e Educacional, 16(1), 71-81. doi: 10.1590/S1413-85572012000100008 
Kreuz, G., \& Franco, M. H. P. (2017). O luto do idoso diante das perdas da doença e do envelhecimento Revisão Sistemática de Literatura. Arquivos Brasileiros de Psicologia, 69(2), 168-186. Recuperado de https://bit.ly/2WsHRSR

Kubler-Ross, E. (2008). Sobre a morte e o morrer: o que os doentes terminais têm para ensinar a médicos, enfermeiras, religiosos e aos seus próprios parentes (9a ed.). São Paulo, SP: Martins Fontes. (Trabalho original publicado em 1969)

Lima, M. J. V. (2019). Uma escuta ética do cuidado na morte e no morrer. Fortaleza, CE: EdUECE.

Lima, M. J. V., \& Andrade, N. M. (2017). A atuação do profissional de saúde residente em contato com a morte e o morrer. Saúde Sociedade, 26(4), 958-972.

Lima, M. J. V., \& Lopes, F. G. (2020). Como comunicar às crianças a morte de um ente querido por Covid-19. Fortaleza, CE: EdUECE.

Lindemann, E. (1944). The symptomatology and management of acute grief. The American Journal of Psychiatry, 101, 141-148. Recuperado de https://bit.ly/3zWNJkQ

Lisbôa, M. L., \& Crepaldi, M. A. (2003). Ritual de despedida em familiares de pacientes com prognóstico reservado. Paidéia, 13(25), 97-109.

Marques, M. (2015). Fatores que impedem a resolução do luto. Psicologia.PT, 1-8. Recuperado de https://bit. 1y/2Y5BJA9

Mazorra, L. (2009). A construção de significados atribuídos à morte de um ente querido e o processo de luto [Tese de Doutorado]. Pontifícia Universidade Católica de São Paulo, São Paulo, SP. Recuperado de https://bit.ly/3zXR57k

Michel, L. H. F, \& Freitas, J. L. (2019). A clínica do luto e seus critérios diagnósticos: possíveis contribuições de Tatossian. Psicologia USP, 30, 1-9. doi: 10.1590/0103-6564e180185

Milstein, C. (Ed.). (2017). Rebellious mourning: the collective work of grief. Chico: Ak Press.

Ministério da Saúde. (2020). Manejo de corpos no contexto do novo coronavírus - Covid-19. Brasília, DF: Ministério da Saúde. Recuperado de https://bit.ly/3D4djGq

Miyazaki, M. C. O. S., \& Teodoro, M. (2020). Tópico 6: luto. Ribeirão Preto, SP: SBP. Recuperado de https://bit.ly/2Y3AeTc

Moore, K., Tulloch, O., \& Ripoll, S. (2020). Key considerations: dying, bereavement and mortuary and funerary practices in the context of Covid-19. Brighton: IDS. Recuperado de https://bit.ly/2WsKUuh

Morand, A., Fabre, A., Minodier, P., Boutin, A., Vanel, N., Bosdure, E., \& Fournier P. E. (2020). Covid-19 virus and children: what do we know? Archives de Pédiatrie, 27(3), 117-118. Recuperado de https://bit.ly/3zXHU6X

Moritz, R. D. (2005). Os profissionais de saúde diante da morte e do morrer. Revista Bioética, 13(2), 51-56. Recuperado de https://bit.ly/3mgU1a2

Noal, D. D. S, Vicente, L. N., Weintraub, A. C. A. D. M., Fagundes, S. M. S, Cabral, K. V., Simoni, A. C. R, . . . Pulino, L. H. C. Z. (2016). Estratégia de saúde mental e atenção psicossocial para afetados da boate Kiss. Psicologia: Ciência \& Profissão, 36(4), 932-945. doi: 10.1590/1982-3703002062016

Oliveira e Silva, A. C. (2014). Conceituando o luto. In F. S. Santos, A. L. Schliemann, \& J. P. C. Solano, Tratado brasileiro sobre perdas e luto (pp. 71-78). São Paulo, SP: Atheneu.

Ornell, F., Schuch, J. B., Sordi, A. O., \& Kessler, F. H. P. (2020). "Pandemic fear" and Covid-19: mental health burden and strategies. Brazilian Journal of Psychiatry, 42(3), 232-235. doi: 10.1590/1516-4446-2020-0008

Parkes, C. M. (1998). Luto: estudos sobre a perda na vida adulta (3a ed.). São Paulo, SP: Summus.

Ramos, H. (2015). Além-túmulo no Facebook: vida após a morte e luto na era digital. Observatório, 9(4), 31-50. Recuperado de https://bit.ly/2ZAu0ef

Rando, T. A., Doka, K. J., Fleming, S., Franco, M. H., Lobb, E. A., Baded, M., .. Steele, R. (2012). A call to the field: complicated grief in the DSM-5. OMEGA - Journal of Death and Dying, 65(4), 251-255. doi: 10.2190/OM.65.4.a

Rumbold, B., \& Aoun, S. (2014). Bereavement and palliative care: a public health perspective. Progress in Palliative Care, 22(3), 131-135.

Sbizera, C. L. (2018). O coping religioso-espiritual no processo de luto em pessoas que perderam entes queridos para o suicídio [Dissertação de mestrado]. Universidade Presbiteriana Mackenzie, São Paulo, SP. Recuperado de https://bit.ly/3os0zWp

Schmidt, B., Crepaldi, M. A., Bolze, D. A. S, Neiva-Silva, L., \& Demenech, L. M. (2020). Impactos na saúde mental e intervenções psicológicas diante da pandemia do Novo Coronavírus(Covid-19).Estudos dePsicologia (Campinas), 37, 1-13. doi: 10.1590/1982-0275202037e200063

Schmidt, B., Gabarra, L. M., \& Gonçalves, J. R. (2011). Intervenção psicológica em terminalidade e morte: relato de experiência. Paidéia, 21(50), 423-430. Recuperado de https://bit.ly/2WwugtM

Shojaei, S. F., \& Masoumi, R. (2020). The importance of mental health training for psychologists in Covid-19 outbreak. Middle East Journal of Rehabilitation and Health Stududies, e102846. Recuperado de https://bit.ly/3ol9toi

Silva, T. C. (2012). Musealização de eventos críticos: análise da tensão entre múltiplas narrativas da dor. In I. Tamaso \& M. F. Lima Filho (Orgs.), Antropologia e patrimônio cultural: trajetórias e conceitos (pp. 497-525). Brasília, DF: ABA.

Solano, J. P. C. (2014). Modelos de luto "normal". In F. S. Santos, J. P. C. Solano, \& A. L. Schliemann (Ed.), Tratado brasileiro sobre perdas e luto (pp. 109-112). São Paulo, SP: Atheneu.

Stroebe, M., \& Schut, H. (2010). The dual process model of coping with bereavement: a decade on. Westport: Omega, 61(4), 273-289. doi: 10.2190/OM.61.4.b.

Taylor, S. (2019). The psychology of pandemics: preparing for the next global outbreak of infectious disease. Newcastle upon Tyne: Cambridge Scholars Publishing.

Wallace, C. L., Wladkowski, S. P., Gibson, A., \& White, P. (2020). Grief during the Covid-19 pandemic: 
considerations for palliative care providers. Journal of Pain and Symptom Management, 60(1), 70-6. Recuperado de https://bit.ly/2WquVwC

Walter, T. (2008). The new public mourning. In M. S. Stroebe, R. O. Hansson, H. Schut, \& W. Stroebe, Handbook of bereavement research and practice: advances in theory and intervention (pp. 241-262). Washington, DC: American Psychological Association.

Walter, T. (2015). New mourners, old mourners: online memorial culture as a chapter in the history of mourning. New Review in Hypermedia and Multimedia, 21(1-2), 10-24. Recuperado de https://bit.ly/3ASS9dT

Wang, S. S., Teo, W. Z., Yee, C. W., \& Chai, Y. W. (2020). Pursuing a good death in the time of Covid-19. Journal of Palliative Medicine, 23(6), 754-755. Recuperado de https://bit.ly/3imrfUi

Weide, J. N., Vicentini, E. C. C., Araujo, M. F., Machado, W. L., \& Enumo S. R. F. (2020). Cartilha para enfrentamento do estresse em tempos de pandemia. Porto Alegre, RS: PUCRS.

Weir, K. (2020, April 1). Grief and Covid-19: mourning our bygone lives. American Psychological Association.
Washington, DC: APA. Recuperado de https://bit. ly/3kWMzl3

Worden, W. J. (2013). Aconselhamento do luto e terapia do luto: um manual para profissionais da saúde mental. São Paulo, SP: Roca.

Worden, J. W. (2018). Grief counseling and grief therapy: a handbook for the mental health practitioner. New York: Springer.

World Health Organization. (2007). Risk reduction and emergency preparedness: World Health Organization six-year strategy for the health sector and community capacity development. Geneva: WHO.

Zhai, Y., \& Du, X. (2020). Loss and grief amidst Covid-19: a path to adaptation and resilience. Brain, Behavior, and Immunity, 87, 80-1. Recuperado de https://bit.ly/3kU9zBm

Recebido: $13 / 08 / 2021$

Aprovado: 24/09/2021 